Article

\title{
Lattice Vibration of Layered GaTe Single Crystals
}

\author{
Tao Wang ${ }^{1,2, *(1)}$, Qinghua Zhao ${ }^{1,2}$, Yaping Miao ${ }^{3}$, Fei Ma ${ }^{3}$, Yong Xie ${ }^{4}$ (i) and Wanqi Jie ${ }^{1,2}$ \\ 1 State Key Laboratory of Solidification Processing, Northwestern Polytechnical University, \\ Xi'an 710072, China; nwpugate@mail.nwpu.edu.cn (Q.Z.); jwq@nwpu.edu.cn (W.J.) \\ 2 Key Laboratory of Radiation Detection Materials and Devices, Ministry of Industry and Information \\ Technology, Xi'an 710072, China \\ 3 State Key Laboratory for Mechanical Behavior of Materials, Xi'an Jiaotong University, Xi'an 710049, China; \\ miaoyp2007@126.com (Y.M.); mafei@mail.xjtu.edu.cn (F.M.) \\ 4 State Key Discipline Laboratory of Wide Band Gap Semiconductor Technology, School of Advanced \\ Materials and Nanotechnology, Xidian University, Xi'an 710071, China; yxie@xidian.edu.cn \\ * Correspondence: taowang@nwpu.edu.cn; Tel.: +86-29-88495414
}

Received: 12 January 2018; Accepted: 30 January 2018; Published: 1 February 2018

\begin{abstract}
The effect of interlayer interaction on in-layer structure of laminar GaTe crystals was studied according to the lattice vibration using micro-Raman analysis. The results were also confirmed by the first principle calculations. Accordingly, the relationship between lattice vibration and crystal structure was established. Ten peaks were observed in the micro-Raman spectra from $100 \mathrm{~cm}^{-1}$ to $300 \mathrm{~cm}^{-1}$. Eight of them fit Raman-active vibration modes and the corresponding displacement vectors were calculated, which proved that the two modes situated at $128.7 \mathrm{~cm}^{-1}$ and $145.7 \mathrm{~cm}^{-1}$ were related to the lattice vibration of GaTe, instead of impurities or defects. Davydov splitting in GaTe was identified and confirmed by the existence of the other two modes, conjugate modes, at $110.7 \mathrm{~cm}^{-1}\left(\Delta \omega=33.1 \mathrm{~cm}^{-1}\right)$ and $172.5 \mathrm{~cm}^{-1}\left(\Delta \omega=49.5 \mathrm{~cm}^{-1}\right)$, indicates that the weak interlayer coupling has a significant effect on lattice vibrations in the two-layer monoclinic unit cell. Our results further proved the existence of two layers in each GaTe unit cell.
\end{abstract}

Keywords: GaTe; lattice vibration; crystal structure; Davydov splitting

\section{Introduction}

The III-VIA semiconductor compounds, composed of Ga and In with S, Se, and Te, exhibits laminar structures with strong in-layer bonds and weak interlayer interactions. These special structures can exhibit unique in-plane and out-of-plane optical and electrical properties [1-3], which apply to potential applications in the field of terahertz radiation emission [4,5], radiation detectors [6,7], and solar cells [8] in the past few years. Most III-VIA layered compounds, such as GaS, GaSe, InSe [9,10], have hexagonal structure with all $\mathrm{M}-\mathrm{M}(\mathrm{M}=\mathrm{Ga}, \mathrm{In})$ bonds perpendicular to the layers, and the main symmetry axis parallel to M-M bonds. However, GaTe has a monoclinic layered structure with $\mathrm{C}_{2 / \mathrm{m}}$ space-group symmetry, where only two third of Ga-Ga bonds lie perpendicular to the layers and the other one lies in the layers [11]. These bonds form chains of twofold rotational symmetry inside the layers along the twofold rotational symmetry axis.

The few-layered two-dimensional GaTe materials with direct band gap were successfully prepared and applied as the channel of photodetectors [12-15], which exhibited a fairly high photoresponsivity of $10^{4} \mathrm{~A} / \mathrm{W}$ and a very fast photoresponse time less than $20 \mathrm{~ms}$ [13], which was much better than other layered materials [16,17]. Such wonderful performance may relate to its unique monoclinic structure, however, the crystal structure of GaTe is still not fully understood.

Raman spectroscopy has been used for the analysis of GaTe crystal structure. Cerdeira et al. [18] studied the Raman spectrum of GaSe, GaTe, and $\mathrm{GaSe}_{1-\chi} \mathrm{Te}_{X}$ in the full composition range, and 
theoretically predicted that the conjugate modes or Davydov splitting should appear in the Raman spectrum due to its special layered structure where each cell contains two layers. However, there are only two barely resolved satellite peaks of GaTe with Davydov Splitting frequency $\Delta \omega=25 \mathrm{~cm}^{-1}$ and $\Delta \omega=32 \mathrm{~cm}^{-1}$ around the peaks $62 \mathrm{~cm}^{-1}$ and $132 \mathrm{~cm}^{-1}$. Irwin et al. [19] studied the long-wavelength vibration modes of monoclinic GaTe by Raman scattering, far-infrared absorption and reflection. They argued that there was no conclusive evidence concerning the existence of conjugate modes or Davydov splitting by comparing Raman and infrared absorption results. The possible explanation given by them was that the unit cell of GaTe might have only one layer. Yamamoto et al. [20] carried out a position-dependent micro-Raman measurement with resolution of $1 \mu \mathrm{m}$ at $18 \mathrm{~K}$. Fourteen Raman active modes, including $11 \mathrm{~A}_{\mathrm{g}}$ and $3 \mathrm{~B}_{\mathrm{g}}$ modes, were identified from the 16 energy peaks, which are consistent with the results presented by Irwin et al. [19]. The two rest peaks located at $178.8 \mathrm{~cm}^{-1}$ and $272.1 \mathrm{~cm}^{-1}$ were determined as the satellite peaks around the Raman shifts at $171.8 \mathrm{~cm}^{-1}$ and $270.5 \mathrm{~cm}^{-1}$, respectively, while they attributed them to experimental conditions, instead of Davydov splitting in GaTe crystals. The essence of the argument on conjugate modes or Davydov splitting in GaTe is that the unit cell structure of GaTe has not been well understood, and the Davydov splitting will be found in a two-layered unit cell, not a single-layered one. Moreover, the origin of the peaks in the range of $120 \mathrm{~cm}^{-1}$ to $145 \mathrm{~cm}^{-1}$ was also in dispute. Irwin et al. [19] attributed these peaks to $\mathrm{TeO}_{2}$ on the surface formed by overheating in crystal growth processes. Cerdeira et al. [18] attributed the peak situated at $143 \mathrm{~cm}^{-1}$ to the particular vibration mode in GaTe crystal structure because of the strong Raman emission in pure GaTe. Most recently, S. Huang et al. [20] carried out a much more detailed analysis about GaTe phonon vibration modes based on first-principles calculations, and they recommended that two peaks at $126 \mathrm{~cm}^{-1}$ and $142 \mathrm{~cm}^{-1}$ might originate from the double-resonant Raman mode which are the combinations of phonon around $60-70 \mathrm{~cm}^{-1}$.

The difference in reported Raman spectra of GaTe indicates the deviation of crystal structures from the perfect crystalline due to the existence of stress, defects or symmetry in as-grown GaTe samples. The quality of GaTe crystals depends on the stoichiometry concentration deviation controlled by vapor pressures of elements $\mathrm{Ga}$ and Te during crystal growth, and other process parameters. This possibility has been explained by Yamamoto et al. [21], based on a simple theoretical analysis.

In this paper, we make an effort to establish the relationship between lattice vibration of GaTe and crystal structure through experimental measurements and first principle calculations. High-quality GaTe single crystal was fabricated using Bridgman method and the first principle calculations will be carried out by using Vienna Ab-initio Simulation Package (VASP) (Materials Design, Vienna, Austria). Influence of weak interlayer interaction on the lattice vibration will be analyzed.

\section{Experiments and Calculations}

Layered GaTe single crystals were grown by the Bridgman method. The samples with mirror-like surfaces were prepared by a razor blade from a cleaved ingot with the typical size of $10 \mathrm{~mm} \times 10 \mathrm{~mm} \times 1 \mathrm{~mm}$. In order to get accurate results, the freshly cleaved samples were immediately used for the experimental observation to avoid the effect coming from surface oxidation.

For the photoluminescence measurements, the samples were attached on a cold copper finger of a closed-cycle cryostat with grease to keep the environment temperature at $10 \mathrm{~K}$. A $20 \mathrm{~mW}$ argon ion laser in the wavelength of $488 \mathrm{~nm}$ with the spot of $1 \mathrm{~mm}$ in diameter was used for excitation of PL spectra, the band position resolution is $\pm 0.3 \mathrm{~nm}$.

Micro-Raman spectroscopy measurements were performed on a Renishaw InVia Laser-Raman Spectrometer (Renishaw, New Mills, United Kingdom) at $300 \mathrm{~K}$. Ar ion laser with the wavelength of $514.5 \mathrm{~nm}$ and a $1 \mu \mathrm{m}$ spot in diameter was used as excitation source. The back-scattering geometry was used for the measurements, which meant the incident light and collections of Raman signals were perpendicular to the surface layers. The resolution of Raman spectrum was $1 \mathrm{~cm}^{-1}$, Raman band positions had the accuracy of $\pm 0.1 \mathrm{~cm}^{-1}$. The energy frequency was above $100 \mathrm{~cm}^{-1}$. 
First-principle calculations were carried out using the density functional theory (DFT) as implemented in MedeA-VASP. The calculation details can be found in our previous paper [22].

\section{Results and Discussion}

Figure 1 shows a typical PL spectrum of GaTe single crystals excited with $2.54 \mathrm{eV}$ laser radiation at $10 \mathrm{~K}$ in the energy region of $1.5-1.8 \mathrm{eV}$. The spectrum consists of three well resolved emission bands, labeled as A, B and C. Band A is weak, and broad with asymmetric Gaussian-shape centered at $1.57 \mathrm{eV}$ and extended from $1.55 \mathrm{eV}$ to $1.60 \mathrm{eV}$. Band $\mathrm{B}$ is a broad band with the peak located at $1.74 \mathrm{eV}$, extending from $1.70 \mathrm{eV}$ to $1.77 \mathrm{eV}$. Band $\mathrm{C}$ has a strong and sharp peak centered at $1.7785 \mathrm{eV}$, with the full-width at half maximum (FWHM) of only $3 \mathrm{meV}$, which is sharper than the reported $\mathrm{GaTe}$ band $[23,24]$. According to the established band gap levels and recombination process in GaTe single crystals $[23,24]$, the band A belongs to donor-acceptor pair (DAP) transitions from a donor level situated at around $80 \mathrm{meV}$ below the conduction band to an acceptor level $150 \mathrm{meV}$ over the valence band. The band $\mathrm{B}$ consists of two peaks located at $1.72 \mathrm{eV}$ and $1.76 \mathrm{eV}$, respectively, formed by the recombination of excitons trapped in two acceptor levels with ionization energies of $110 \mathrm{meV}$ and $150 \mathrm{meV}$. The band C corresponds to free-exciton (FX) with the thermal activation energy at around $18 \mathrm{meV}$. The weak DAP bands and the sharp strong FX bands indicate the low defect concentration and perfect crystal structure of our GaTe samples.

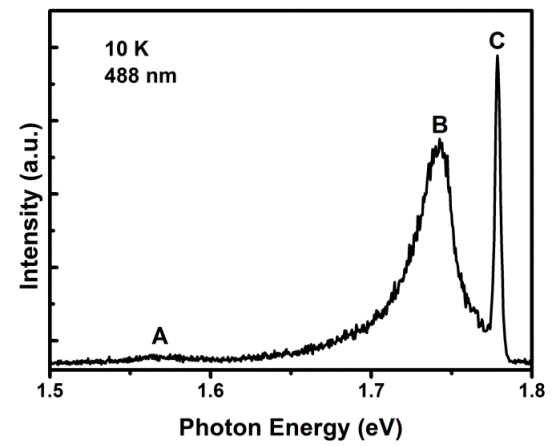

Figure 1. Low-temperature (10 K) photoluminescence (PL) spectrum of GaTe single crystals.

A typical Raman spectrum of GaTe single crystals from $100 \mathrm{~cm}^{-1}$ to $300 \mathrm{~cm}^{-1}$ Raman shift at an excitation wavelength of $514.5 \mathrm{~nm}$ is shown in Figure 2. The incident light power is $5 \mathrm{~mW}$. Ten energy peaks are observed. Among them, the eight located at $109.6 \mathrm{~cm}^{-1}, 114.5 \mathrm{~cm}^{-1}, 151.7 \mathrm{~cm}^{-1}, 161.6 \mathrm{~cm}^{-1}$, $175.2 \mathrm{~cm}^{-1}, 209.6 \mathrm{~cm}^{-1}, 267.1 \mathrm{~cm}^{-1}, 281.7 \mathrm{~cm}^{-1}$ match previous reports for monoclinic GaTe single crystals [18-20,24]. However, there is still controversy over the interpretation of the other two situated at $129.4 \mathrm{~cm}^{-1}$ and $141.8 \mathrm{~cm}^{-1}$.

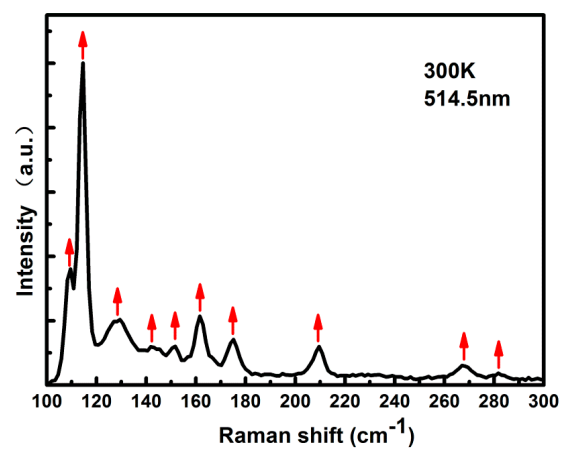

Figure 2. Raman spectrum of GaTe single crystals at $300 \mathrm{~K}$ from $100 \mathrm{~cm}^{-1}$ to $300 \mathrm{~cm}^{-1}$. 
To understand the Raman spectrum, the phonon dispersions were calculated based on GGA-PBE optimized structure. For the monoclinic structure of GaTe single crystals with $\mathrm{C}_{2 / \mathrm{m}}$ symmetry, the long-wavelength optical phonons can be analyzed by group theory and described by the irreducible representations as

$$
\Gamma \equiv 12 \mathrm{~A}_{\mathrm{g}}+6 \mathrm{~A}_{\mathrm{u}}+6 \mathrm{~B}_{\mathrm{g}}+12 \mathrm{~B}_{\mathrm{u}} .
$$

The acoustic modes are $\left(A_{u}+2 B_{u}\right)$. The modes $\left(5 A_{u}+10 B_{u}\right)$ are infrared active, and all the $A_{g}$ and $\mathrm{B}_{\mathrm{g}}$ are Raman active. There are 18 distinct Raman-active modes at the zone center.

The calculated results are depicted in Figure S1. There are 36 lines corresponding to 3 acoustic modes, 15 infrared-active modes and 18 Raman-active modes. The acoustic modes suggest that isolated GaTe crystals are dynamically stable. 8 Raman shifts were calculated by Raman-active phonon frequencies according to the measurement results in the range of $100 \mathrm{~cm}^{-1}$ to $300 \mathrm{~cm}^{-1}$. The comparison between measurements and calculations is given in Table 1. The irreducible representations of $160.4 \mathrm{~cm}^{-1}$ and $172.5 \mathrm{~cm}^{-1}$ are both $\mathrm{B}_{\mathrm{g}}$, and the others are $\mathrm{A}_{\mathrm{g}}$. The small deviations can be explained by internal stress and discrepancies of surface conditions. Additionally, the displacement vectors for the 8 calculated frequencies are given by MedeA-VASP analysis, as shown in Figure 3. The corresponding direction of axis is shown in Figure S2.

Table 1. Comparison of Raman-active frequencies between our measurements and calculations in the range of $100 \mathrm{~cm}^{-1}-300 \mathrm{~cm}^{-1}$.

\begin{tabular}{|c|c|c|c|c|}
\hline Feature Peak & $\begin{array}{c}\text { Irreducible } \\
\text { Representation }\end{array}$ & $\begin{array}{l}\text { Calculated Raman-Active } \\
\text { Phonon Frequencies } \\
\left(>100 \mathrm{~cm}^{-1}\right)\end{array}$ & $\begin{array}{l}\text { Raman-Active Phonon } \\
\text { Frequencies by Our } \\
\text { Measurements }\left(\mathrm{cm}^{-1}\right)\end{array}$ & $\begin{array}{l}\text { The Measurement Results of } \\
\text { Yamamoto et al. }\left(>100 \mathrm{~cm}^{-1}\right)\end{array}$ \\
\hline 1 & $\mathrm{Ag}$ & 110.7 & $109.6,114.5$ & $111.3,116.2$ \\
\hline 3 & $\mathrm{Ag}$ & 145.6 & 141.8 & - \\
\hline 4 & $\mathrm{Ag}$ & 153.4 & 151.7 & 155.2 \\
\hline 5 & $\mathrm{Bg}$ & 160.4 & 161.6 & 166.6 \\
\hline 8 & $\mathrm{Ag}$ & 268.5 & 267.1 & 270.5 \\
\hline 9 & $\mathrm{Ag}$ & - & 281.7 & 287.5 \\
\hline
\end{tabular}

* Reference [21].

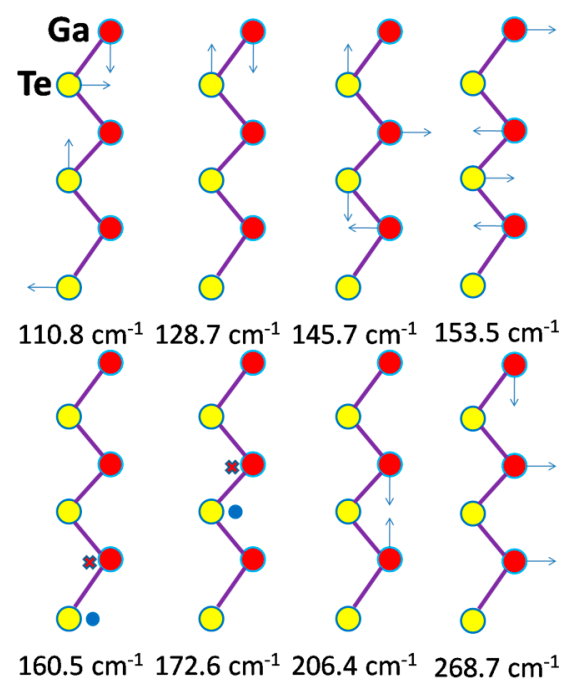

Figure 3. Displacement vectors corresponding to eight Raman vibration modes of GaTe single crystals.

From our calculations, there are two Raman-active phonon frequencies centered at $128.6 \mathrm{~cm}^{-1}$ and $145.6 \mathrm{~cm}^{-1}$ in the range of $120 \mathrm{~cm}^{-1}$ to $150 \mathrm{~cm}^{-1}$, in agreement with our experimental measurements. 
The irreducible representations of them are all $\mathrm{Ag}_{\mathrm{g}}$. When $\mathrm{Ga}$ and Te atoms vibrate along the direction of displacement vectors shown in the Figure 3, the polarization ratio and dipole moment of Te-Ga bonds will be all changed, due to the absence of symmetric centers in Ga-Te bonds. Therefore, the two optical phonons should be observed in Raman spectra and infrared reflection spectra simultaneously. Shared with Abdullaev et al.'s reference [25], two infrared reflection active phonons located at $130 \mathrm{~cm}^{-1}$ and $142 \mathrm{~cm}^{-1}$ were found. The intensity of the peak located at $143 \mathrm{~cm}^{-1}$ increases as Te concentration grows in $\mathrm{GaSe}_{1-\chi} \mathrm{Te}_{\mathrm{X}}$, becoming very strong in pure GaTe. Apparently, the two peaks at $128.7 \mathrm{~cm}^{-1}$ and $145.7 \mathrm{~cm}^{-1}$ may originate from the Raman active vibration modes, but not defects. On the other hand, the layered GaTe single crystal structures are dominated by interlayer coupling and covalent bands within the layers. The couplings between the layers are known to be weaker compared to the intralayer force. Thus, the interlayer couplings should have effects upon the lattice vibration frequencies. Because the unit cell contains two layers, the normal modes occur in pairs with both layers vibrating in or out of phase. These are called conjugate modes and would be split by the interlayer interactions. Both were observed in Raman spectra. This splitting was named Davydov splitting. Evidences for Davydov splitting have been reported for GaSe [2], $\mathrm{MoS}_{2}$ [26] and other layered semiconductors [27].

The support of our assumption on Davydov splitting is the two measured vibration frequencies at $109.6 \mathrm{~cm}^{-1}$ and $114.5 \mathrm{~cm}^{-1}$ located on the two sides of the calculated frequency $110.7 \mathrm{~cm}^{-1}$. Two similar peaks $\left(111.3 \mathrm{~cm}^{-1}, 116.2 \mathrm{~cm}^{-1}\right)$ were also found by Yamamoto et al. [21], which are deemed to two vibration modes. Here we estimate the frequency of the interlayer vibration by the equation:

$$
\Delta \omega=\left(\omega_{1}^{2}-\omega_{2}^{2}\right)^{\frac{1}{2}}
$$

where $\omega_{1}$ and $\omega_{2}$ are the frequencies of the doublet components, and $\Delta \omega$ represents frequency of interlayer vibrations, which is $33.12 \mathrm{~cm}^{-1}$ for the frequency of interlayer coupling, in good agreement with our calculated low-frequency interlayer coupling at $37.5 \mathrm{~cm}^{-1}$. Another experimental data pair by Yamamoto et al. [21], fully consistent with Davydov splitting, are $171.8 \mathrm{~cm}^{-1}$ and $178.8 \mathrm{~cm}^{-1}$, corresponding to the Raman active vibration mode located at $172.5 \mathrm{~cm}^{-1}$. The value of $\Delta \omega=49.5 \mathrm{~cm}^{-1}$ is close to the calculated frequency at $50.9 \mathrm{~cm}^{-1}$. More interestingly, we also found the other two conjugated mode pairs by the S. Huang et al.'s [20] calculation results. In their Supplementary Information (Figure S3), for the calculated mode pairs, $25\left(A_{u} \omega=173.6 \mathrm{~cm}^{-1}\right)$ and $23\left(\mathrm{~A}_{\mathrm{u}} \omega=168.8 \mathrm{~cm}^{-1}\right), 24\left(\mathrm{~B}_{\mathrm{g}} \omega=172.7 \mathrm{~cm}^{-1}\right)$ and $22\left(\mathrm{Bg}_{\mathrm{g}} \omega=167.6 \mathrm{~cm}^{-1}\right)$, with the interlayer coupling frequency $40.5 \mathrm{~cm}^{-1}$ and $41.7 \mathrm{~cm}^{-1}$, respectively, were observed. The interlayer coupling frequency derived by Equation (1) shows very good agreement with the first-principles calculated results, $40.5 \mathrm{~cm}^{-1}$ and $41.8 \mathrm{~cm}^{-1}$. More noteworthy is that the vibration direction of each individual atom is the same $\left(0^{\circ}\right)$ or opposite $\left(180^{\circ}\right)$, the only difference being in the force constant.

For the Davydov splitting in layered GaTe single crystals, the van der Waals interaction and long-range Coulombic force by electrostatic interactions should be taken into considerations. A classical model for coupled harmonic oscillators was employed for interpretations and the Raman-active vibration frequency $\omega$ could be estimated by the harmonic oscillation equation:

$$
\omega=\frac{1}{2 \pi} \sqrt{\frac{k}{\mu}}
$$

where $k$ is restoring force constant and $\mu$ is the equivalent mass of the coupled harmonic oscillators, which could be obtained by

$$
\mu^{-1}=\mathrm{M}_{\mathrm{A}}^{-1}+\mathrm{M}_{\mathrm{B}}^{-1}
$$

$\mathrm{M}_{\mathrm{A}}$ and $\mathrm{M}_{\mathrm{B}}$ are the mass of the oscillators. For Ga-Ga or Ga-Te bonds in GaTe crystal, the coupling frequency $\omega$ is only determined by the restoring force constant $k$. When the interlayer interactions and intralayer interactions in GaTe single crystals are in phase or out of phase, different $k$ corresponding 
to different vibration frequencies would appear and Davydov splitting should be observed in GaTe Raman spectra.

Therefore, the fact that there are two layers in each GaTe unit cell could be explained by the existence of the conjugated modes. The second proof of our assignment is the stacking direction of the layered structure in GaTe crystals. Previous measurements show that the index of crystallographic plane of GaTe surface is (-210) [28], which indicates that the stacking direction could be determined in the unit cell shown in the Figure 4. It is apparent that there are two layers in each unit cell of GaTe.

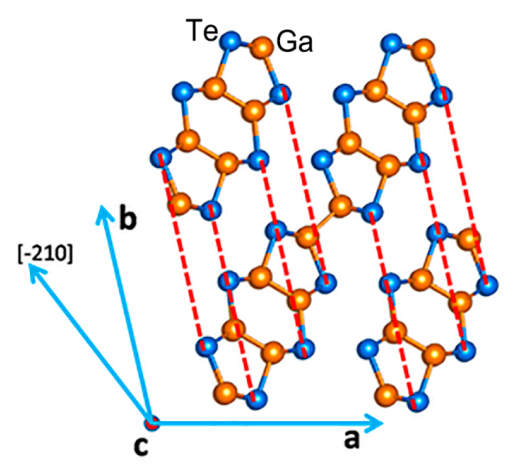

Figure 4. The stacking direction of the layers in GaTe crystal structure.

\section{Conclusions}

Based on high-quality crystalline GaTe single crystals obtained by Bridgman method, the lattice vibration properties were studied by micro-Raman spectroscopy observation and first-principles calculations. Ten peaks were observed, among which eight Raman-active vibration modes were identified from $100 \mathrm{~cm}^{-1}$ to $300 \mathrm{~cm}^{-1}$ by comparing with the calculated phonon dispersion. The existence of Davydov splitting was proved in GaTe. Two conjugate modes at $110.7 \mathrm{~cm}^{-1}$ $\left(\Delta \omega=33.1 \mathrm{~cm}^{-1}\right)$ and $172.5 \mathrm{~cm}^{-1}\left(\Delta \omega=49.5 \mathrm{~cm}^{-1}\right)$ were determined, which supported the existence of two layers in each GaTe unit cell. Our results showed that the lattice vibration of GaTe was jointly decided by crystal structure and interlayer interaction.

Supplementary Materials: The following are available online at http:/ /www.mdpi.com/2073-4352/8/2/74/s1, Figure S1: Calculated phonon dispersion. Figure S2: The direction of axis in GaTe.

Acknowledgments: This work was supported by the National Key R\&D Program of China (No. 2016YFB0402405, No. 2016YFF0101301) and the National Natural Science Foundations of China (No. 51672216). This work was supported by the research fund of the State Key Laboratory of solidification processing (NWPU, 94-QZ-2014, SKLSP201612); The Fundamental Research Funds for the Central Universities (No. G2016KY0104, No. 3102016ZY011); Work was also supported by the 111 Project of China (No. B08040). The work was also supported by State Key Laboratory for Mechanical Behaviour of Materials. Qinghua Zhao acknowledges the grant from the China Scholarship Council (CSC) under No. 201700290035. Yong Xie is grateful for financial support from the China Scholarship Council (CSC) under No. 201706965048.

Author Contributions: Tao Wang, Wanqi Jie and Qinghua Zhao performed the experiments and wrote the paper. Yaping Miao and Fei Ma performed the first-principles calculations. Yong Xie helped to prepare the samples.

Conflicts of Interest: The authors declare no conflicts of interests.

\section{References}

1. Gupta, V.; Srivastava, V. Opto-electronic properties of gallium chalcogenides. J. Phys. Chem. Solids 1981, 42, 1071-1077. [CrossRef]

2. Hayek, M.; Brafman, O.; Lieth, R. Splitting and coupling of lattice modes in the layer compounds GaSe, GaS, and $\mathrm{GaSe}_{\mathrm{X}} \mathrm{S}_{1-\mathrm{X}}$. Phys. Rev. B 1973, 8, 2772. [CrossRef]

3. Zhu, Z.; Cheng, Y.; Schwingenschlögl, U. Topological phase transition in layered GaS and GaSe. Phys. Rev. Lett. 2012, 108, 266805. [CrossRef] [PubMed] 
4. Xu, G.; Sun, G.; Ding, Y.J.; Zotova, I.B.; Mandal, K.C.; Mertiri, A.; Pabst, G.; Roy, R.; Fernelius, N.C. Investigation of terahertz generation due to unidirectional diffusion of carriers in centrosymmetric GaTe crystals. IEEE J. Sel. Top. Quant. 2011, 17, 30-37. [CrossRef]

5. Mandal, K.C.; Kang, S.H.; Choi, M.; Chen, J.; Zhang, X.-C.; Schleicher, J.M.; Schmuttenmaer, C.A.; Fernelius, N.C. III-VI chalcogenide semiconductor crystals for broadband tunable THz sources and sensors. IEEE J. Sel. Top. Quant. 2008, 14, 284-288. [CrossRef]

6. Reshmi, P.; Kunjomana, A.; Chandrasekharan, K.; Meena, M.; Mahadevan, C. Structural, electrical and mechanical properties of GaTe for radiation detector applications. Int. J. Soft Comput. Eng. 2011, 1, $228-232$.

7. Nelson, A.J.; Conway, A.M.; Sturm, B.; Behymer, E.; Reinhardt, C.; Nikolic, R.; Payne, S.; Pabst, G.; Mandal, K. $\mathrm{X}$-ray photoemission analysis of chemically treated GaTe semiconductor surfaces for radiation detector applications. J. Appl. Phys. 2009, 106, 023717. [CrossRef]

8. Mandal, K.C.; Das, S.; Krishna, R.; Muzykov, P.G.; Ma, S.; Zhao, F. Surface passivation of p-GaTe layered crystals for improved p-GaTe/n-InSe heterojunction solar cells. MRS Online Proc. Libr. Arch. 2010, 1268, 1268-EE1202-1210. [CrossRef]

9. McCanny, J.; Murray, R. The band structures of gallium and indium selenide. J. Phys. C Solid State Phys. 1977, 10, 1211. [CrossRef]

10. Olguin, D.; Rubio-Ponce, A.; Cantarero, A. Ab initio electronic band structure study of III-VI layered semiconductors. Eur. Phys. J. B 2013, 86, 350. [CrossRef]

11. Schlüter, M.; Camassel, J.; Kohn, S.; Voitchovsky, J.; Shen, Y.; Cohen, M.L. Optical properties of GaSe and $\mathrm{GaS}_{\mathrm{X}} \mathrm{Se}_{1-\mathrm{X}}$ mixed crystals. Phys. Rev. B 1976, 13, 3534. [CrossRef]

12. Hu, P.; Zhang, J.; Yoon, M.; Qiao, X.-F.; Zhang, X.; Feng, W.; Tan, P.; Zheng, W.; Liu, J.; Wang, X. Highly sensitive phototransistors based on two-dimensional GaTe nanosheets with direct bandgap. Nano Res. 2014, 7, 694-703. [CrossRef]

13. Liu, F.; Shimotani, H.; Shang, H.; Kanagasekaran, T.; Zolyomi, V.; Drummond, N.; Fal'ko, V.I.; Tanigaki, K. High-sensitivity photodetectors based on multilayer GaTe flakes. ACS Nano 2014, 8, 752-760. [CrossRef] [PubMed]

14. Wang, Z.; Xu, K.; Li, Y.; Zhan, X.; Safdar, M.; Wang, Q.; Wang, F.; He, J. Role of Ga vacancy on a multilayer GaTe phototransistor. ACS Nano 2014, 8, 4859-4865. [CrossRef] [PubMed]

15. Wang, Z.; Safdar, M.; Mirza, M.; Xu, K.; Wang, Q.; Huang, Y.; Wang, F.; Zhan, X.; He, J. High-performance flexible photodetectors based on GaTe nanosheets. Nanoscale 2015, 7, 7252-7258. [CrossRef] [PubMed]

16. Buscema, M.; Island, J.O.; Groenendijk, D.J.; Blanter, S.I.; Steele, G.A.; van der Zant, H.S.; Castellanos-Gomez, A. Photocurrent generation with two-dimensional van der Waals semiconductors. Chem. Soc. Rev. 2015, 44, 3691-3718. [CrossRef] [PubMed]

17. Xie, C.; Mak, C.; Tao, X.; Yan, F. Photodetectors based on two-dimensional layered materials beyond graphene. Adv. Funct. Mater. 2016, 27, 1603886. [CrossRef]

18. Cerdeira, F.; Meneses, E.; Gouskov, A. Splittings and correlations between the long-wavelength optical phonons in the layer compounds GaSe, GaTe, and GaSe $\mathrm{Te}_{1-\mathrm{X}}$. Phys. Rev. B 1977, 16, 1648. [CrossRef]

19. Irwin, J.; Clayman, B.; Mead, D. Long-wavelength phonons in GaTe. Phys. Rev. B 1979, 19, 2099. [CrossRef]

20. Huang, S.; Tatsumi, Y.; Ling, X.; Guo, H.; Wang, Z.; Watson, G.; Puretzky, A.A.; Geohegan, D.B.; Kong, J.; Li, J.; et al. In-plane optical anisotropy of layered gallium telluride. ACS Nano 2016, 10, 8964. [CrossRef] [PubMed]

21. Yamamoto, A.; Syouji, A.; Goto, T.; Kulatov, E.; Ohno, K.; Kawazoe, Y.; Uchida, K.; Miura, N. Excitons and band structure of highly anisotropic GaTe single crystals. Phys. Rev. B 2001, 64, 035210. [CrossRef]

22. Zhao, Q.; Wang, T.; Miao, Y.; Ma, F.; Xie, Y.; Ma, X.; Gu, Y.; Li, J.; He, J.; Chen, B. Thickness-induced structural phase transformation of layered gallium telluride. Phys. Chem. Chem. Phys. 2016, 18, 18719-18726. [CrossRef] [PubMed]

23. Güder, H.; Abay, B.; Efeoğlu, H.; Yoğurtçu, Y. Photoluminescence characterization of GaTe single crystals. J. Lumin. 2001, 93, 243-248. [CrossRef]

24. Zubiaga, A.; Garcıa, J.; Plazaola, F.; Muñoz-Sanjosé, V.; Martınez-Tomás, M. Recombination processes in unintentionally doped GaTe single crystals. J. Appl. Phys. 2002, 92, 7330-7336. [CrossRef]

25. Abdullaev, G.; Vodopyanov, L.; Allakhverdiev, K.; Golubev, L.; Babaev, S.; Salaev, E.Y. Raman spectra of $\alpha$-GaTe single crystals. Solid State Commun. 1979, 31, 851-855. [CrossRef] 
26. Ghosh, P.N.; Maiti, C. Interlayer force and Davydov splitting in 2H-MoS . Phys. Rev. B 1983, $28,2237$. [CrossRef]

27. Kuroda, N.; Nishina, Y. Davydov splitting of degenerate lattice modes in the layer compound GaS. Phys. Rev. B 1979, 19, 1312. [CrossRef]

28. Balitskii, O.; Jaeckel, B.; Jaegermann, W. Surface properties of GaTe single crystals. Phys. Lett. A 2008, 372, 3303-3306. [CrossRef] 